\title{
Methods for polymer-stabilization of molecular orientation in LC- waveguiding structures
}

\author{
Katarzyna A. Rutkowska, ${ }^{*}$ Miłosz Chychłowski, \\ Faculty of Physics, Warsaw University of Technology, Koszykowa 75, 00-662 Warszawa
}

Received March 27, 2018; accepted March 31, 2018; published March 31, 2018

\begin{abstract}
In this letter we present a new way for constructing and developing LC-based waveguiding structures. Specifically, liquid crystalline material enriched with a monomer in the proper proportions is implemented in our approach and waveguide geometry can be created in the LC cell thanks to the photo-polymerization process allowing for LC molecules to be suspended in a desired position and orientation. The latter can be enforced by external fields, and UV-irradiation can be performed through the mask of desired geometry. In this way, regions with different molecular textures (e.g. planar and homeotropic) and thus waveguiding structures can be easily created within an LC layer. The main idea of this letter is not only to show the proof-of-concept of polymer-imposed LC stabilization for such planar optical devices but to discuss differences in the methodology of their practical realization.
\end{abstract}

Liquid crystals, their derivatives and compounds obtained by adding specific components like e.g. dyes, nanoparticles or momoners are commonly used in photonic applications [1-5]. Specifically, polymerdispersed and stabilized LCs are promising candidates for practical realization of new optical elements and devices in photonic systems, while offering wider functionalities than those achieved in pure LC $[1,3,4,6]$.

As it has been shown in our previous works [7-9], polymer-stabilized LCs can be successfully applied for fabrication of liquid crystalline waveguiding structures with the use of the photo-polymerization process. For this purpose a typical nematic liquid crystal (e.g. 6CHBT, E7 or PCB NLC) is combined with a small amount of mixture composed of a monomer and an UV-sensitive activator. In our case, the RM257 monomer (1,4-Bis-[4-(3acryloyloxypropyloxy)benzoyloxy]-2-methylbenzene)

[10] has been mixed with a DMPA photo-initiator $(2,2-$ dimethoxy-2-phenylacetophenone) [11], with their percentage weight less than $10 \%$ in the LC-monomer mixture.

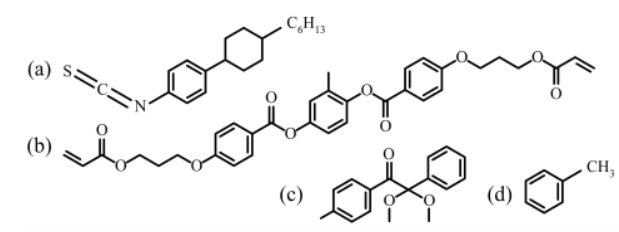

Fig. 1. Chemical structures of 6CHBT NLC (a), RM257 monomer (b) and DMPA photo-initiator (c). Toluene (d) has been used as a transferring liquid for RM257 and DMPA to NLC in one of the fabrication procedures.

$$
\text { *E-mail: kasia@if.pw.edu.pl }
$$

The RM257 monomer consists of rod-like molecules (Fig. 1b) and is usually photo-polymerized with polymer networks solidifying the direction of LC alignment. DMPA is typically used as a photo-initiator in dental light-cured composites. The monomer and photo-initiator applied here are commercially available from Merck and Sigma-Aldrich, respectively.

The main advantage of LC-based waveguiding structures, when compared to analogical ones obtained in other materials, is the possibility of changing optical properties and characteristics after their fabrication. In principle, molecular reorientation obtained under the influence of an external electric field or electromagnetic wave in the LC layer, may be used as an efficient method to control light propagation. Such tunability and possibility of creating the waveguides of almost any arbitrary geometry (determined e.g. by the shape of an amplitude mask applied during the UV-illumination process) is the reason why the proposed polymerstabilized waveguiding structures may find many potential applications as LC-based elements and devices for integrated optics. On the other hand, there are several advantages of our technique of creating controllable waveguiding structures in liquid crystalline materials when compared to other LC-based waveguides, which are: (i) low power of a propagating light beam (i.e. there is no need to use soliton-like beams to create the waveguide channel [12]); (ii) possible uniformity of orienting layers in the whole area of the LC cell, not like in Ref. [13]; (iii) possibility of easily created complex structures and desired refractive index contrast by selective irradiation without any special electrode structure or modified orienting layer [8].

The most important parameters to be adjusted and controlled when fabricating the waveguiding structures of adequate quality are: composition of the LC-monomer mixture, UV light power density and time interval between successive illuminations [8,9]. As we show in this letter, also the way in which the LC-monomer mixture is prepared plays an important role in the fabrication process.

The simplest idea to create waveguiding structures in the LC material enriched with a monomer is to reorient LC molecules by an external electric field and irradiate 
the selected area of the LC layer with UV light to polymerize the monomer and "freeze" the molecules in a specific orientation. In addition, two different approaches may be applied in which LC molecules in the remaining area are or are not polymer-stabilized. In the second option, the molecules go back to their original orientation when the LC sample is not biased. Taking LC cells with the initial planar orientation enforced by orienting layers (Fig. 2a) and assuming that a bias-induced reorientation takes place in the transverse $x-y$ plane, the molecular arrangement may be described by the orientation angle $\theta$ (Fig. 2e) and thus effective refractive indices for TE- and TM-like polarization is defined as: $n_{\text {eff TM/TE }}(\theta)=n_{o} n_{e}$. $\cdot\left[\left(n_{e / o}^{2}-n_{o / e}^{2}\right) \cos ^{2} \theta+n_{o / e}^{2}\right]^{-1 / 2}$, where $n_{o}$ and $n_{e}$ are the ordinary and extraordinary refractive indices, respectively.

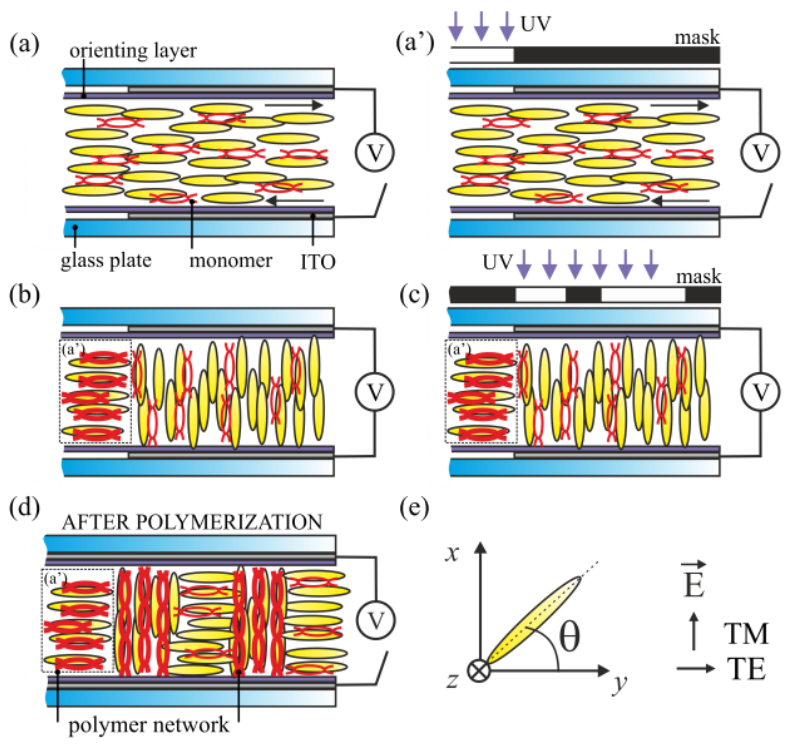

Fig. 2. Scheme of the proposed LC-based waveguiding structure preparation procedure: (a) infiltration of LC cell with an LC-monomer mixture, (a') UV-induced photo-polymerization of the selected area, (b) LC molecular reorientation with the use of an external electric field, (c) photo-polymerization through the amplitude mask of desired geometry, (d) creation of a stable molecular arrangement within the LC layer after unbiasing the sample. Please note that the step (a') is optional when the polymer-stabilized planar orientation in a part of the LC sample is required. Panel (e) shows possible molecular orientation in the $x-y$ plane, described by the orientation angle $\theta$. TE- and TM-like polarizations are defined assuming that the light beam propagates along the $z$-axis direction.

A schematic representation of the fabrication process is shown in Fig. 2, where the main steps of this procedure are presented. The idea behind using an LC-monomer mixture in planar LC-based devices is to create a desired photonic structure with a specific spatial distribution of LC orientation and thus an effective refractive index by the photo-polymerization process performed in selected areas with a specified LC molecular arrangement. If UVirradiation is performed with the use of an amplitude mask, after the procedure is completed, not only the polymerized LC may be again reoriented, while the LC molecular orientation in the whole polymer-stabilized region is fixed indefinitely.

The main difficulties to be faced in the fabrication process are: (i) suitable conditions of the photopolymerization process (including exposition time to UVlight of specific intensity) and (ii) composition and uniformity of the LC-monomer mixture itself. Because of the existence of a polymer in the LC cell, one expects light scattering to be increased. In this context, the minimum weight concentration of monomer to stabilize NLC and to get molecular arrangement control should be reached [8].

Table 1. Compositions of two different LC-monomer mixtures compared in this letter.

\begin{tabular}{|c|c|c|c|c|c|c|}
\hline \multicolumn{3}{|c|}{ Mixture \#1 } & \multicolumn{4}{c|}{ Mixture \#2 } \\
\hline NLC & RM257 & DMPA & NLC & RM257 & DMPA & toluene \\
\hline \multicolumn{5}{|c|}{ wt. \% } \\
\hline 94.9 & 4.15 & 0.95 & 95.3 & 4.14 & 0.56 & + \\
\hline
\end{tabular}

Table 2. Parameters of UV-illumination procedures performed to photopolymerize LC-monomer mixtures, where "exposition" is the exposition time, "blank" is the period of time between successive illuminations and "repetition" is the number of repetitions in a cure cycle. Light intensity of the Dymax BlueWave ${ }^{\circledR} 75$ UV curing spot lamp has been kept at a level of $250 \mathrm{~mW} / \mathrm{cm}^{2}$ in all cases.

\begin{tabular}{|c|c|c|c|c|c|}
\hline \multicolumn{3}{|c|}{ Mixture \#1 } & \multicolumn{3}{c|}{ Mixture \#2 } \\
\hline exposition & blank & repetitions & exposition & blank & repetitions \\
\hline $0.4 \mathrm{~s}$ & $10 \mathrm{~s}$ & $3 \div 6$ & $0.3 \mathrm{~s}$ & $60 \mathrm{~s}$ & $4 \div 5$ \\
\hline
\end{tabular}

The composition of two different LC-monomer mixtures as well as parameters of their UV-illuminations are summarized in Table 1 and Table 2, respectively. It is worth noting that two different preparation methods for mixing an LC material with a monomer are presented in this letter, leading to creation of stable distinct orientations of liquid crystalline molecules within an LC cell. Specifically, toluene was used as a solvent and liquid carrier for powder components in Mixture \#2, to enhance the uniformity of an LC-monomer mixture. In this case, the RM257 monomer and the DMPA photo-initiator (both in the powder form) were dissolved in toluene, with the latter making 90 wt. \% of the (toluene + RM257 + DMPA) mixture. It was then added to NLC in the isotropic phase and vortex mixed (for 10 minutes) and placed in the ultrasonic bath (for 20 minutes). Toluene was evaporated from the mixture which was kept at a temperature of $5^{\circ} \mathrm{C}$ higher than the clearing point of NLC and this process took about 40 hours. The appraisement of its completeness was performed based on observation how mixture returned to the nematic phase after it was cooled down to room temperature. Mixture \#1 was prepared in such a way that the RM257 monomer and the DMPA photo-initiator were completely dissolved in the host NLC in the isotropic phase by vortex mixing and sonification. Such prepared mixtures were capillary filled into the $50 \mu \mathrm{m}$-thick cells, which were assembled from two pieces 

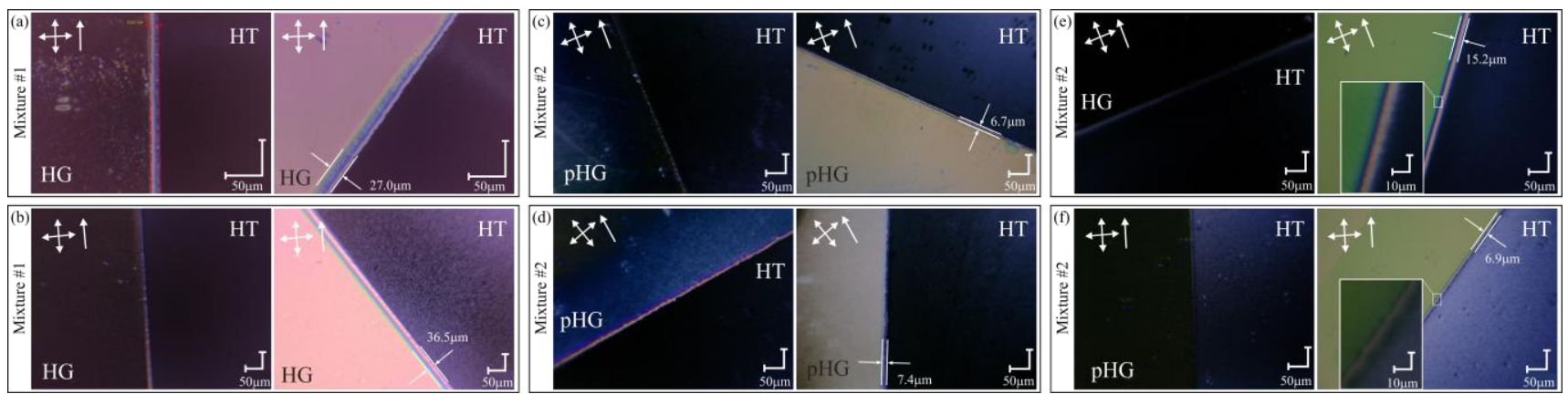

Fig. 3. Photos of the top views (y-z plane) of LC layers taken with the use of a polarized light digital microscope for Mixture \#1 (a, b) and Mixture \#2 (c-f). Directions of the polarizer and analyzer axis (crossed double-ended arrows), as well as initial planar orientation in the LC sample (single arrow) are indicated. Regions with different textures, i.e. homeotropic (HT) and planar (HG: non-cured, pHG: UV-cured), are observed and the thickness of the boundary between them is measured (with use of digital microscope, as shown on the left photo in Panel (a)).

of ITO-coated glass substrates with their inner surfaces coated with rubbed polyimide layers giving a planar alignment. Their quality was checked under a polarized light microscope. During the first UV exposure, an electric field was applied to the sample (please note that the ITO electrode does not cover the whole area of a glass substrate) and a photomask was used to define the desired pattern. The value of applied AC voltage was high enough to get molecular reorientation from the initial planar (HG) to the homeotropic (HT) one. Then, UV-light is illuminated on the cell while voltage is applied on the cell for a certain time. We have checked that the polymerization rate is a key issue to make the cured texture uniform. A good approach to improve the uniformity of the polymerized area and control the LC arrangement efficiently is utilizing multiple cure cycles [8]. In the second option, the sample was exposed to a UV light source again without voltage applied to completely polymerize the remaining material.

The samples fabricated were characterized using a white light polarizing microscope. Figure 3 shows how their quality changes depending on the way they were produced. Apart from two different mixtures to be used, three scenarios of UV-irradiation procedure were applied: (i) only the region with an HT texture was photopolymerized (Figs. 3a, b, e); (ii) HG and HT alignment were irradiated at the same time (Figs. 3d, f); (iii) planarly oriented sample was illuminated and after the part with the electrodes was uncovered when the bias was on and reoriented part with HT orientation was then illuminated in the similar way (Fig. 3c). The fundamental features of making high-quality regions of a desired molecular orientation when observed between crossed polarizers were: (i) phase difference between the cured and noncured/additionally cured areas of different molecular textures; (ii) straightness and thickness of the fringe boundary lines between such two regions; (iii) texture uniformity of the cured area(s). Results obtained for Mixture \#1 were characterized by high inhomogeneities and the presence of scattering centers in both polymerized (cured) and non-cured regions, as well as irregular (creased) and wide boundary between them. These conditions were getting even worse when the number of cure cycles were too high (Fig. 3b). Toluene used as a solvent to mix the monomer in the host NLC ensured the uniformity of textures and reduced the thickness of the boundary line at least twice (Fig. 3e). The latter may be thinned even more when the region with a planar texture is also UV-irradiated (Figs. 3c, 3d, 3f). It means that a relatively wide intersection between $\mathrm{HG}$ and $\mathrm{HT}$ alignment in Fig. 3e is related to the gradual reorientation of LC-molecules cross-linked in the HT alignment and to not that of polymer-stabilized HG.

In conclusion, we have presented two different methods possibly leading to creation of polymer-assisted LC-based waveguiding structures for integrated optics. We have demonstrated that the way in which the LC-monomer mixture is obtained and then UV-illuminated to get polymer-stabilized structure is crucial for further practical applications in functional photonics.

Authors would like to acknowledge the support by the Polish National Science Center (NCN) under the grant no. DEC-2013/11/B/ST7/04330.

\section{References}

[1] Q. Li, Liquid crystals beyond displays: chemistry, physics, and applications (John Wiley \& Sons, 2012).

[2] J.P. Lagerwall, G. Scalia, Current App. Phys. 12, 1387 (2012).

[3] A. Ciferri, Polymer liquid crystals (Elsevier, 2012).

[4] D. Yang, Fundamentals of liquid crystal devices (John Wiley \& Sons, 2014).

[5] L. Vicari, Optical applications of liquid crystals (CRC press, 2016).

[6] I. Dierking, Adv Mater 12, 167 (2000).

[7] K. Rutkowska, M. Chychłowski, M. Kwaśny, I. Ostromęcka, J. Piłka, U. Laudyn, Opto-Electr. Rev. 25, 118 (2017).

[8] K A. Rutkowska, M. Chychłowski, U.A. Laudyn, Proc. SPIE 10325, 1032506 (2017).

[9] B. Turowski, K.A. Rutkowska, Phot. Lett. Poland 9, 82 (2017),

[10] H. Ren, Y. Lin, S. Wu, Opt. Commun. 261, 296 (2006).

[11] V. Mucci and C. Vallo, J Appl Polym Sci 123, 418 (2012).

[12] U.A. Laudyn, M. Kwaśny, K. Jaworowicz, K.A. Rutkowska, M.A. Karpierz, G. Assanto, Phot. Lett. Poland 1, 7 (2009).

[13] M.S. Chychłowski, S. Ertman, E. Nowinowski-Kruszelnicki, T.R. Woliński, Mol. Cryst. Liquid Cryst. 553, 127 (2012). 